\title{
miRNA: the new frontier in cancer medicine
}

\author{
"We are now in the middle of a critical and exciting time in miRNA-based cancer \\ research and anticancer therapeutic development."
}

Keywords: cancer stem cell $\approx$ microRNA $\approx$ resistance $\approx$ therapeutics

Although cancer is a genetic disease, epigenetic alternations (e.g., methylation, histone modification, noncoding RNA) are understood to play a key role in tumorigenesis. Such alterations enhance the ability of tumor cells to adopt to various growth conditions and to their unique tumor microenvironment.

Approximately $2 \%$ of transcripts code for mRNA templates for protein synthesis, leaving a large percentage of noncoding RNAs (lncRNAs, miRNAs and piRNAs) many of which are highly conserved. The regulatory roles of these noncoding RNAs are just beginning to emerge. miRNAs are a class of small noncoding RNAs (e.g., siRNA, piRNA) with crucial regulatory function $[1,2]$. miRNAs modulate protein expression by promoting RNA degradation, inhibiting mRNA translation and, in some cases, affecting transcription. Although miRNA-mediated mRNA degradation occurs in mammals, most mammalian miRNAs are thought to repress target gene expression at the translational level via imperfect base pairing to the 3'-untranslated regions of their target mRNAs $[1,3,4]$. Such translational regulation provides the cell with a more precise, immediate and energy-efficient way of controlling the expression of protein as it induces rapid changes in protein synthesis [5], without excess transcriptional activation and subsequent steps in mRNA processing. In addition, translational control of gene expression has the advantage of being readily reversible, providing the cell with great flexibility in responding to various genotoxic and cytotoxic stresses. The relevance of miRNA in cancer was revealed a decade later when a link was found between miR-15, miR-16 and chronic lymphocytic leukemia [6]. The impact of miRNAs in cancer has become a new frontier in cancer research.

In addition to the use miRNA mimics or anti-miRs as novel anti-cancer therapeutics alone, they have great potential to be used in combination with current chemotherapeutic compounds or radiation therapy. Resistance to chemotherapy and radiation therapy is one of the major reasons for the failure of clinical cancer management. Many factors contributed to the resistance mechanism and miRNAs have been shown to play major roles [7]. Posttranscriptional and translational controls mediated by miRNAs are crucial for tumors to develop resistance quickly by modulating protein synthesis in response to genotoxic stresses during anticancer chemotherapy and/or radiation therapy. As a result, modulating miRNA expression may offer new therapeutic strategies to overcome drug resistance in tumors. Chemotherapeutic agents (e.g., 5-fluorouracil) are ineffective in eliminating the slow proliferating cancer stem cells and, thus, novel approaches may help to overcome this issue. It is important to discover genes and pathways that are responsible for the resistance mechanism. Due to the broad influence of miRNA in gene expression, we believe that miRNAs may offer new insights into the resistance mechanism. In some cases, miRNAs may contribute to the development of resistance while in others they may help to overcome resistance. We have recently reported that miR-140 is overexpressed in $\mathrm{CD} 133^{+\mathrm{Hi}} \mathrm{CD} 44^{\mathrm{HH}}$ colon cancer stem-like cells and by reducing its expression, we can sensitize chemoresistant CD $133^{+\mathrm{Hi}} \mathrm{CD} 44^{+\mathrm{Hi}}$ colon cancer stem-like cells to 5-fluorouracil treatment [8].

Tumor cells often lose their ability to undergo apoptosis due to defects in both intrinsic and extrinsic pathways. A number of miRNAs have been recently discovered to modulate key apoptosis protein targets (e.g., BCL-2, XIAP and SIRT-1). BCL-2 is an anti-apoptotic protein and is overexpressed in many cancer types. Several strands of miRNA have been reported to regulate BCL-2, increasing apoptosis. In gastric cancer cell lines high in BCL-2, miR-34 was observed to decrease BCL-2, increasing apoptosis and chemosensitivity [9]. Similar effects on apoptosis were found in primary chronic lymphocytic

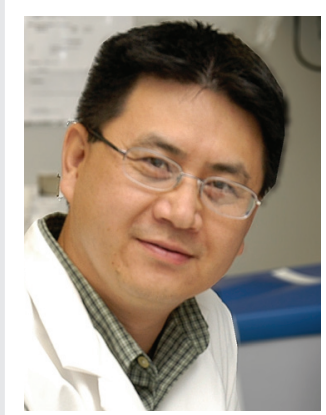

Jingfang Ju

Author for correspondence: Translational Research Laboratory, Department of Pathology, BST, L-9, Room 185, Stony Brook Medicine, Stony Brook University, Stony Brook, NY II794, USA Tel.: + | 63। 4443598 E-mail: jingfang.ju@ stonybrookmedicine.edu

Jingting Jiang

Department of Tumor Biotherapy, The Third Affiliated Hospital of Soochow University, Changzhou, PR China

\section{Andrew Fesler}

Department of Pathology, Stony Brook Medicine, Stony Brook University, Stony Brook, NY I1794, USA 
leukemia CLL cells with miR-181 targeting BCL-2 [10]. These are just some examples of how miRNA can engage in modulating apoptosis in cancer and have therapeutic potential.

\section{"The relevance of miRNA in cancer was revealed a decade later when a link was found between miR-15, miR-16 and chronic lymphocytic leukemia. The impact of miRNAs in cancer has become a new frontier in cancer research."}

However, it is a difficult task to realize the dream of miRNA-based therapeutics and we are facing a number of challenges. Challenges, such as stability, toxicity, distribution and specificity to target tumor cells, are similar to those faced by other nucleic acid-based compounds. With the extensive efforts from both academia and industry, a number of solutions are now available to bring miRNA therapeutics closer to patients. With the issue of stability, extensive efforts have been made to modify miRNAs for enhanced stability by various modifications of nucleotides, such as $2^{\prime}-O$-methyl and $2^{\prime}$-O-methoxyethyl [11], anti-miRs, as well as with 3 '-end-cholesterol conjugation $[11,12]$ and locked nucleic acid [13-15]. Such modifications have been demonstrated to enhance anti-miR stability and affinity to miRNA. However, care has to be taken to avoid off-target effects due to extensive modifications.

As for the in vivo delivery strategies, a number of approaches have been attempted such as the use of lentiviral vectors. Lentiviral vectors offer the advantage of stable gene therapy due to their integration into the genome. It has also been observed that lentiviral vectors offer greater transduction efficiency than other viral vectors in lung cancer [16]. However, there remains the concern that viral integration could disrupt the host genome, although some work has indicated that lentiviral integration poses a limited threat to genomic integrity. There is also concern regarding the development of an immune response to the lentiviral vector, which could limit the effectiveness of treatment. Other viral vectors for miRNA delivery are adenoviral -based vectors. Adenoviral-based vectors are episomal compared with lentiviral vectors but also have toxicity-related issues associated with immune response to the virus, as well as the concern of overcome existing immunity.
Adeno-associated virus has also been reported to be effective at delivering miRNA in mouse models of liver cancer [17]. Nonviral delivery mechanisms have also demonstrated potential for therapeutic delivery of miRNA. These include liposomes, which have been shown to effectively deliver miRNA to cancer cells both locally and systemically in mouse xenograft models $[13,18]$. In addition, nanoparticles made of gold or synthetic polymers can effectively deliver miRNA when administered systemically in mouse xenograft models, leading to reduced tumor growth [19]. Recently an exosomal-based miRNA delivery has been reported with great potential as effective therapeutic delivery systems [20]. Exosomal delivery, offers a unique opportunity for miRNA delivery due to the fact that this system is naturally used in cellular communication, probably contributing to its stability in circulation. Exosomes can also be modified so that they can target delivery to a particular cell type. While exosome delivery seems promising, more work needs to be carried out, to make the production and isolation of exosomes an efficient process. There is also concern that an immune response could be mounted against exosomes unless they are harvested from immune-compatible cells.

\section{"Many factors contributed to the resistance mechanism and miRNAs have been shown to play major roles."}

We are now in the middle of a critical and exciting time in miRNA-based cancer research and anticancer therapeutic development. In the next 5-10 years, the efforts from both academia and industry will pay off to realize the dream of having multitargeted miRNA-based anticancer therapy to benefit patients. miRNA-based diagnosis will also impact patient care by offering personalized diagnosis and tumor-tailored therapy. We remain optimistic about the miRNA-based diagnosis and future medicine in cancer.

Financial \& competing interests disclosure $J J u$ is funded by the NIH. The authors have no other relevant affiliations or financial involvement with any organization or entity with a financial interest in or financial conflict with the subject matter or materials discussed in the manuscript apart from those disclosed.

No writing assistance was utilized in the production of this manuscript. 


\section{References}

1 Lee RC, Feinbaum RL, Ambros V. The C. elegans heterochronic gene lin- 4 encodes small RNAs with antisense complementarity to lin-14. Cell75(5), 843-854 (1993).

2 Wightman B, Ha I, Ruvkun G.

Posttranscriptional regulation of the heterochronic gene lin-14 by lin- 4 mediates temporal pattern formation in $C$. elegans. Cell 75(5), 855-862 (1993).

3 Pillai RS, Bhattacharyya SN, Artus CG et al. Inhibition of translational initiation by Let-7 microRNA in human cells. Science 309(5740), 1573-1576 (2005).

4 Ruvkun G. Clarifications on miRNA and cancer. Science 311(5757), 36-37 (2006).

5 Dony C, Kessel M, Gruss P. Posttranscriptional control of myc and p 53 expression during differentiation of the embryonal carcinoma cell line F9. Nature 317(6038), 636-639 (1985).

6 Calin GA, Dumitru CD, Shimizu M et al. Frequent deletions and down-regulation of micro-RNA genes miR15 and miR16 at 13 q14 in chronic lymphocytic leukemia. Proc. Natl Acad. Sci. USA 99(24), 15524-15529 (2002).

7 Yu F, Yao H, Zhu P et al. Let-7 regulates self renewal and tumorigenicity of breast cancer cells. Cell 131(6), 1109-1123 (2007).
8 Song B, Wang Y, Xi Y et al. Mechanism of chemoresistance mediated by miR-140 in human osteosarcoma and colon cancer cells. Oncogene 28(46), 4065-4074 (2009).

9 Ji Q, Hao X, Meng Y et al. Restoration of tumor suppressor miR-34 inhibits human p53-mutant gastric cancer tumorspheres. BMC Cancer 8, 266 (2008).

10 Zhu DX, Zhu W, Fang C et al. miR-181a/b significantly enhances drug sensitivity in chronic lymphocytic leukemia cells via targeting multiple anti-apoptosis genes. Carcinogenesis 33(7), 1294-1301 (2012).

11 Park JK, Kogure T, Nuovo GJ et al. miR-221 silencing blocks hepatocellular carcinoma and promotes survival. Cancer Res. 71(24), 7608-7616 (2011).

12 Krutzfeldt J, Rajewsky N, Braich R et al. Silencing of microRNAs in vivo with 'antagomirs'. Nature 438(7068), 685-689 (2005).

13 Wiggins JF, Ruffino L, Kelnar K et al. Development of a lung cancer therapeutic based on the tumor suppressor microRNA-34. Cancer Res. 70(14), 5923-5930 (2010).

14 Obad S, dos Santos CO, Petri A et al. Silencing of microRNA families by seedtargeting tiny LNAs. Nat. Genet. 43(4), 371-378 (2011).
15 Kurreck J, Wyszko E, Gillen C, Erdmann VA. Design of antisense oligonucleotides stabilized by locked nucleic acids. Nucleic Acids Res. 30(9), 1911-1918 (2002).

16 Chen C, Akerstrom V, Baus J, Lan MS, Breslin MB. Comparative analysis of the transduction efficiency of five adeno associated virus serotypes and VSV-G pseudotype lentiviral vector in lung cancer cells. Virol. J. 10, 86 (2013).

17 Kota J, Chivukula RR, O’Donnell KA et al. Therapeutic microRNA delivery suppresses tumorigenesis in a murine liver cancer model. Cell 137(6), 1005-1017 (2009).

18 Rai K, Takigawa N, Ito S et al. Liposomal delivery of microRNA-7-expressing plasmid overcomes epidermal growth factor receptor tyrosine kinase inhibitor-resistance in lung cancer cells. Mol. Cancer Ther. 10(9), 1720-1727 (2011).

19 Chen Y, Zhu X, Zhang X, Liu B, Huang L. Nanoparticles modified with tumor-targeting $s c F v$ deliver siRNA and miRNA for cancer therapy. Mol. Ther. 18(9), 1650-1656 (2010).

20 Valadi H, Ekstrom K, Bossios A, Sjostrand M, Lee JJ, Lotvall JO. Exosome-mediated transfer of mRNAs and microRNAs is a novel mechanism of genetic exchange between cells. Nat. Cell Biol. 9(6), 654-659 (2007). 